\title{
Impactos da COVID-19 sobre os profissionais de saúde no contexto pandêmico: uma revisão integrativa da literatura
}

\author{
Impacts of COVID-19 on health professionals during the pandemic context: an integrative \\ literature review
}
Impactos de COVID-19 sobre los profesionales de la salud durante el contexto de la pandemia: una revisión bibliográfica integradora

Francielle Bianca Moreira de Mesquita ${ }^{1 *}$, Pedro César Morato Filho ${ }^{1}$, Ruan Teixeira Lessa ${ }^{1}$, Lucas Augusto Niess Soares Fonseca ${ }^{1}$, Davi Martins Vidal ${ }^{1}$, Danilo José Martins de Souza ${ }^{1}$, Tayná Beato Ferreira $^{1}$, Vitória Leite Silva ${ }^{1}$, Galileu Bitencourt Batista ${ }^{1}$, Nathália Barbosa do Espírito Santo Mendes ${ }^{1,2}$.

\section{RESUMO}

Objetivo: Relacionar os impactos da COVID-19 sobre os profissionais de saúde no contexto pandêmico. Métodos: Realizou-se uma revisão literária nas seguintes bases de dados científicos da área médica: SciELO, PUBMED e BVS-Bireme. Como critérios de inclusão, foram filtrados apenas artigos originais, revisões, diretrizes e recomendações de órgãos científicos oficiais publicados em $2020 \mathrm{com}$ os descritores "Pessoal de Saúde", "Infecções por Coronavirus", "Impactos na Saúde", "Pandemias", e excluídos artigos anteriores ao ano de 2020. Resultados: Foram relatados os números de profissionais de saúde mortos e infectados através dos artigos revisados e pelos órgãos governamentais de saúde dos países consultados, além da constatação dos impactos relacionados à disponibilidade de material hospitalar, afecções psicossociais e surgimento ou agravo de doenças pré-estabelecidas. Considerações finais: Os profissionais de saúde representam um grupo de grande vulnerabilidade diante da atual pandemia de COVID-19. Os impactos mais relevantes se concentraram na escassez de Equipamentos de Proteção Individual (EPIs), falta de apoio de instituições e autoridades públicas, carência de suporte psicológico e o risco de contágio que eleva sensações de medo e pânico generalizados nessas pessoas.

Palavras-chave: Pessoal de saúde, Infecções por coronavirus, Impactos na saúde, Pandemias.

\begin{abstract}
Objective: Relate the impacts of COVID-19 on health professionals in the pandemic context. 'Methods: A literary review was carried out in the following scientific databases in the medical field: SciElo, PUBMED and BVS-Bireme. As inclusion criteria, only original articles, reviews, guidelines and recommendations from official scientific bodies published in 2020 with the descriptors "Health Personnel", "Coronavirus Infections", "Impacts on Health", "Pandemics" were filtered and articles prior to 2020 were excluded. Results: The numbers of health professionals died and infected were reported through the reviewed articles and by the governmental health agencies of the countries consulted, in addition to the verification of the impacts related to the availability of hospital supplies, psychosocial disorders and appearance or aggravation of pre-established diseases. Final considerations: Health professionals represent a highly vulnerable group in the face of the current COVID19 pandemic. The most relevant impacts were concentrated on the scarcity of Personal Protective Equipment
\end{abstract}

${ }^{1}$ Centro Universitário Presidente Antônio Carlos (UNIPAC-JF), Juiz de Fora - MG.

*E-mail: franbmoreira@gmail.com

2 Universidade Federal de Juiz de Fora (UFJF), Juiz de Fora - MG.

SUBMETIDO EM: 6/2020

ACEITO EM: 7/2020

PUBLICADO EM: 10/2020

REAS/EJCH | Vol.12(10) | e4398 | DOI: https://doi.org/10.25248/reas.e4398.2020 Página 1 de 11 
(PPE), lack of support from public institutions and authorities, lack of psychological support and the risk of contagion that raises feelings of generalized fear and panic in these people.

Keywords: Health personnel, Coronavirus infections, Impacts on health, Pandemics.

\section{RESUMEN}

Objetivo: Relacionar los impactos de COVID-19 sobre los profesionales de la salud en el contexto de la pandemia. Métodos: Se realizó una revisión literaria en las siguientes bases de datos científicos en el campo médico: SciElo, PUBMED y BVS-Bireme. Como criterios de inclusión, sólo se filtraron artículos originales, revisiones, directrices y recomendaciones de organismos científicos oficiales publicados en 2020 con los descriptores "Personal de salud", "Infecciones por coronavirus", "Impactos en la salud", "Pandemias" y se excluyeron artículos anteriores a 2020. Resultados: El número de profesionales de la salud fallecidos e infectados fue obtenido a través de los artículos revisados y por las agencias gubernamentales de salud de los países consultados, además de verificados los impactos relacionados con la disponibilidad de suministros hospitalarios, trastornos psicosociales y la apariencia o agravamiento de enfermedades preestablecidas.

Consideraciones finales: Los profesionales de la salud representan un grupo altamente vulnerable frente a la actual pandemia de COVID-19. Los impactos más relevantes se concentraron en la escasez de equipo de protección personal (EPP), falta de apoyo de las instituciones públicas y autoridades, falta de apoyo psicológico y el riesgo de contagio que aumenta los sentimientos generalizados de miedo y pánico en estas personas.

Palabras-clave: Personal de salud, Infecciones por coronavirus, Impactos en la salud, Pandemias.

\section{INTRODUÇÃO}

Os coronavírus são vírus de RNA envelopados, de origem zoonótica, transmitidos por via aérea; e encontrados em pássaros, seres humanos e outros mamíferos. Há alguns meses, seis espécies de coronavírus eram agentes conhecidos para doenças em seres humanos, sendo quatro desses vírus predominantes e causadores de sintomas respiratórios (229E, OC43, NL63 e HKU1). As outras duas espécies de coronavírus são responsáveis por causar Síndrome Respiratória Aguda Grave (SARS-CoV) e Síndrome Respiratória no Oriente Médio (MERS-CoV) (KOH D, 2020; MARK A, et al., 2020).

Contudo, em 9 de janeiro de 2020, a Organização Mundial da Saúde (OMS) divulgou uma declaração sobre o conjunto de casos da COVID-19 e declarou que as autoridades chinesas fizeram uma determinação de um novo coronavírus, identificado em uma pessoa hospitalizada com pneumonia em Wuhan, província de Hubei na China central. O vírus foi inicialmente referido como 2019-nCoV e posteriormente renomeado como SARS-CoV-2 pela OMS, em 12 de fevereiro de 2020 (KOH D, 2020; ITELMAN E, et al., 2020).

No dia 11 de março de 2020, foi declarada pela OMS, a pandemia do novo coronavírus (SARS-CoV-2). O relatório mais recente do órgão, indica que o número de mortos pelo vírus ao redor do mundo subiu para quase 605.000, dados representativos do dia 20 de julho de 2020. Enquanto a quantidade de casos de COVID-19 confirmados oficialmente aumentou para cerca de 14.500 .000 casos (KAWCHUK, et al., 2020; WORLD HEALTH ORGANIZATION, 2020).

A Itália teve um envolvimento precoce e severo, com uma disseminação crítica da infecção e um número muito alto de vítimas. A transmissão de pessoa para pessoa ocorre principalmente por meio de gotículas respiratórias e contato. $O$ período de incubação varia entre 4 a 14 dias. $O$ espectro de sintomas respiratórios pode alterar de leve a grave, dependendo estritamente da idade do paciente e das comorbidades subjacentes (LICCIARDI F, et al., 2020).

No que tange ao Brasil, a taxa de contaminação tem crescido rapidamente. No dia 29 de fevereiro de 2020 , foi diagnosticado o paciente-zero no país. Já em 29 de abril, dois meses depois, o número de casos em um único dia já era de 6.267, com 464 óbitos. Em 29 de junho de 2020, o número de casos confirmados em um 
único dia já somava 24.052, com 692 óbitos. E, seguindo essa sequência de aumentos, segundo dados do dia 20 de julho de 2020, o número acumulado de pessoas infectadas no país atingiu aproximadamente 2.100.000, enquanto a quantidade de mortes totais se elevou para quase 80.000 , fato esse que evidencia a grande quantidade de brasileiros vítimas dessa pandemia (WORLD HEALTH ORGANIZATION, 2020; MINISTÉRIO DA SAÚDE DO BRASIL, 2020).

Em épocas de surto, os sistemas de saúde podem ser caracterizados como locais conturbados e caóticos, quando um grande número de pacientes chega às unidades de saúde locais para atendimento. $O$ aspecto mais importante de estar preparado é planejar com antecedência. Por isso, o conceito de "triagem direta" foi desenvolvido para responder a vários tipos de cenários de crise, como epidemias de vírus e catástrofes. A triagem direta é o processo de determinar a condição dos pacientes antes que eles cheguem aos serviços de saúde. Assim, a telemedicina direta ao consumidor pode permitir que os pacientes se conectem com seu médico à distância, além disso, as intervenções através da Digital Health ou Saúde Digital permitem que os profissionais de saúde combatam a pandemia global, mesmo que estejam praticando medidas de autoisolamento ou trabalhando remotamente. A telemedicina proporciona a redução dos riscos de contaminação e tem demonstrado ser uma ferramenta essencial ao proporcionar atendimento médico por meio de plataformas especializadas em assistência remota. (MOAZZAMI B, et al., 2020).

Durante pandemias, o mundo enfrenta uma paralisação ou desaceleração nas atividades diárias e os indivíduos são encorajados a implementar o distanciamento social, a fim de reduzir as interações entre as pessoas, consequentemente, a possibilidade de novas infecções. Entretanto, os profissionais de saúde costumam seguir condutas contrárias, devido a sua prestação de serviços e mesmo com o uso de diretrizes que prezam pela redução da possibilidade de contaminação desses trabalhadores, tornou-se inevitável o aumento das chances de transmissão por SARS-CoV-2. Na Coréia, médicos e enfermeiros foram infectados enquanto prestavam cuidados; em um único instituto, mais de 10 médicos ou enfermeiros relataram ter contraído COVID-19 enquanto prestavam assistência ao paciente (ORNELL F, et al., 2020; JIN YH, et al., 2020; HUH S, 2020; BASILE C, et al., 2020).

Apoiado no estudo de Delgado D et al. (2020); esse risco aumentado aos profissionais de saúde já foi observado em epidemias de doenças altamente infecciosas, como Ebola ou SARS, devido ao contato com os pacientes, fluidos corporais contaminados e acesso reduzido ao uso de Equipamentos de Proteção Individual (EPIs). Nesse contexto, os EPIs podem reduzir o risco, uma vez que atuam como barreiras físicas contra o patógeno.

Porém, não está claro qual o tipo de EPI protege mais ou qual a melhor maneira de aplicar ou remover o equipamento e ainda como treinar profissionais de saúde para usá-lo, sendo essas reponsabilidades direcionadas à diretrizes oriundas das autoridades de saúde locais (VERBEEK JH, et al., 2020; LITTLE P, et al., 2020; KISELY S, et al., 2020).

Devido ao aumento exponencial da demanda por assistência médica, eles enfrentam longos turnos de trabalho, geralmente com poucos recursos e infraestrutura precária; e necessidade imperativa de utilizar EPIs que podem causar desconforto físico e dificuldade em respirar, além de eczemas. Isso pode levá-los ao isolamento do ambiente familiar, mudança de rotina e restrição de suas redes de apoio social. Esses fatores resultam em diferentes níveis de pressão psicológica, que podem desencadear sentimentos de solidão e desamparo ou uma série de estados emocionais disfóricos, como estresse, irritabilidade, distúrbios do sono, fadiga mental e desespero. A sobrecarga de trabalho e os sintomas relacionados ao estresse tornam profissionais de saúde especialmente vulneráveis ao sofrimento psicológico (LAN J, et al., 2020; RANNEY ML, et al., 2020; ORNELL F, et al., 2020; KHAN S, et al., 2020; MOAZZAMI B, et al., 2020; GREENBERG N, et al., 2020).

Em suma, devido à pandemia, milhões de pessoas estão em quarentena, a fim de minimizar a transmissão do coronavírus, enquanto os profissionais de saúde se preparam para fazer exatamente o oposto. Eles atuam em clínicas e hospitais, colocando-se em alto risco com a COVID-19. Números da Comissão Nacional de Saúde da China mostram que mais de 3.300 profissionais de saúde foram infectados no início de março e, 
até o final de fevereiro, pelo menos 22 haviam morrido. Na Itália, $20 \%$ dos profissionais de saúde, foram infectados e alguns morreram. No Irã, pelo menos 40 profissionais de saúde vieram a falecer. Isso demonstra a grande capacidade multiplicativa do vírus e a necessidade de se atentar a esse público que é de extrema importância no combate à atual pandemia instalada (MOAZZAMI B, et al., 2020; LANCET T, 2020).

O objetivo desse trabalho, portanto, é relacionar os impactos da COVID-19 sobre os profissionais de saúde no contexto pandêmico.

\section{MÉTODOS}

O desenvolvimento desta revisão integrativa foi fundamentado a partir de um levantamento de dados da literatura, publicados em 2020. As bases de dados científicos utilizados foram, SciELO, PUBMED e BVSBireme, além de sites de órgãos sanitários oficiais, como a OMS e o Ministério da Saúde.

Nas buscas, os seguintes descritores, em língua portuguesa e inglesa, foram considerados: "profissionais de saúde" e "COVID-19". Recorreu-se aos operadores lógicos "AND", "OR", "AND NOT" para combinação dos descritores e termos utilizados para rastreamento das publicações.

Através desse método de busca e abordando as recomendações PRISMA (Principais Itens para Relatar Revisões sistemáticas e Meta-análises), foram identificadas, a princípio, 277 publicações potencialmente elegíveis para integrar esta revisão.

Em seguida, distinguiram-se os artigos que atenderam aos seguintes critérios de inclusão: (a) bibliografias eletrônicas compatíveis com os descritores enumerados acima; (b) cronologia a partir do ano 2020; (c) textos completos e resumos do tipo tese, artigos originais, revisões, instruções normativas e portarias de órgãos científicos oficiais, obtidos nas bases de dados científicos da área médica supracitados; (d) consideração apenas por textos que relacionam os profissionais de saúde à pandemia de COVID-19. Optou-se por não incluir artigos anteriores ao ano de 2020, estudos que abordassem a COVID-19 sem enfoque nos profissionais de saúde e manuscritos sem rigor metodológico.

Após a primeira análise, com avaliação dos títulos, 221 artigos foram considerados selecionáveis para a próxima fase desta revisão, que consistiu na leitura dos resumos. Após a realização do parecer dos resumos, as referências que pareciam integrar os critérios de inclusão e exclusão foram lidas na íntegra e elencados 86 deles. Ao final, 14 estudos foram eleitos.

$\mathrm{Na}$ avaliação, foram observados os seguintes aspectos: amostra (dimensionamento adequado, envolvendo seleção conveniente de participantes; instrumentos (critérios de avaliação); relevância estatística dos estudos; periódico no qual o artigo foi publicado (classificados conforme os critérios Qualis); relação dos impactos da COVID-19 sobre os profissionais de saúde no contexto pandêmico, conforme a (Figura 1). 
Figura 1 - Identificação e seleção dos artigos sobre os impactos da COVID-19 sobre os profissionais de saúde no contexto pandêmico para a revisão integrativa, 2020.

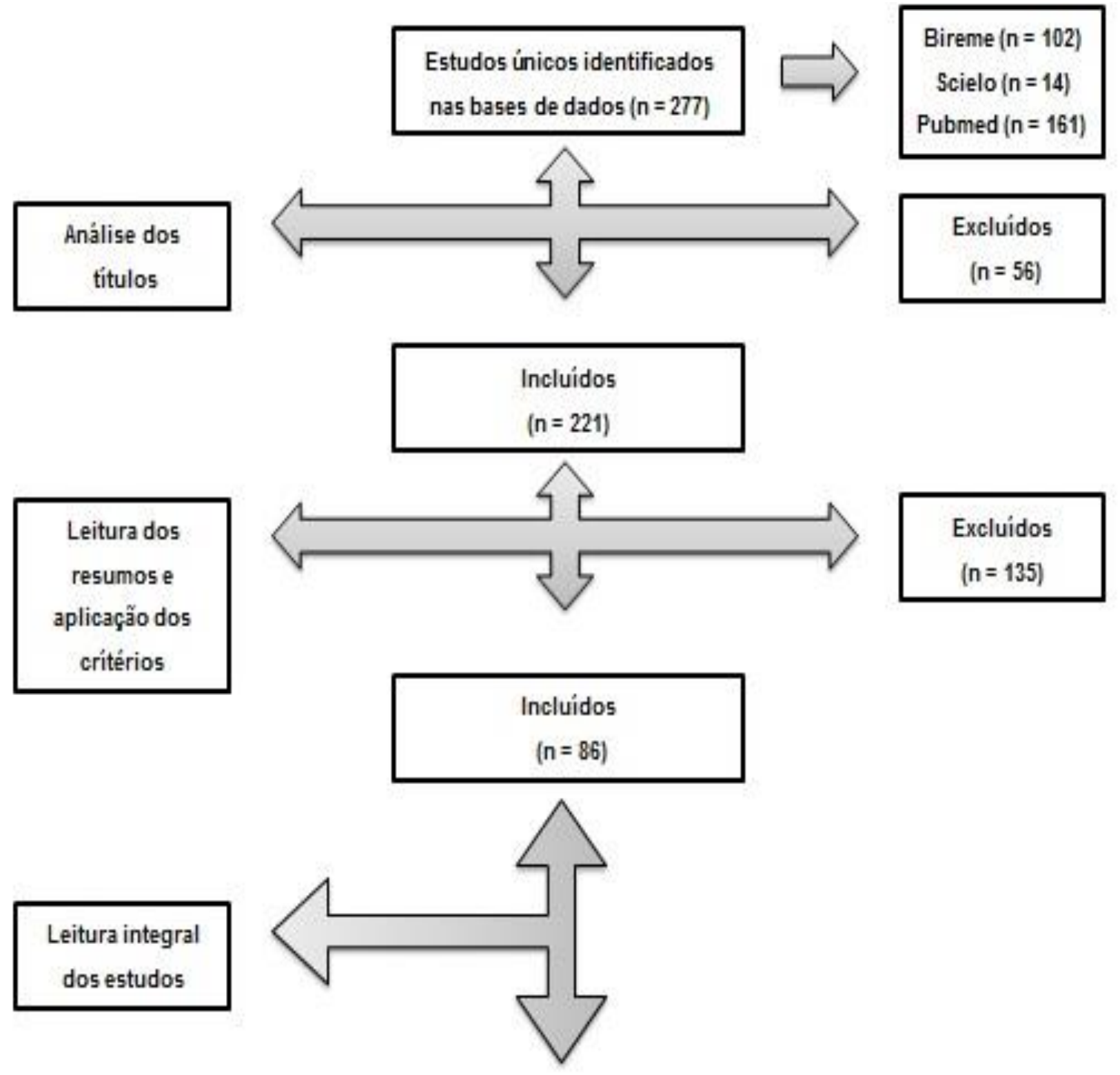

$$
\begin{aligned}
& \text { Incluidos na revisão } \\
& \qquad(n=14)
\end{aligned}
$$

Fonte: de Mesquita FBM, et al., 2020.

\section{RESULTADO E DISCUSSÃO}

A seguir são apresentadas informações gerais sobre os 14 estudos incluídos e publicados no ano de 2020. Nove manuscritos foram divulgados em periódicos classificados como Qualis Nacional A2 ou superior. Os estudos utilizaram como instrumento de pesquisa questionários específicos para a coleta de dados, arquivos hospitalares, escalas e relatórios. A composição das amostras quanto ao $\mathrm{n}$ amostral, incluiu profissionais de saúde, apenas (Tabela 1). 
Tabela 1 - Apresentação detalhada dos estudos incluídos na revisão, por fonte, periódico, Qualis-CAPES, $n$ amostral, instrumento e local de publicação. Juiz de Fora - MG, 2020.

\begin{tabular}{|c|c|c|c|c|c|}
\hline Fonte & Periódico & $\begin{array}{l}\text { Qualis- } \\
\text { CAPES }\end{array}$ & $\begin{array}{c}\mathbf{N} \\
\text { amostral }\end{array}$ & Instrumento & Local \\
\hline $\begin{array}{l}\text { Tostmann A, } \\
\text { et al.,2020 }\end{array}$ & Eurosurveillance & $\mathrm{A} 1$ & 1.247 & Questionário online & $\begin{array}{l}\text { Países } \\
\text { Baixos/ } \\
\text { Europa }\end{array}$ \\
\hline $\begin{array}{l}\text { Misra A, } \\
2020\end{array}$ & $\begin{array}{l}\text { Diabetology \& metabolic } \\
\text { syndrome }\end{array}$ & B1 & 1716 & Questionário & China/ Ásia \\
\hline $\begin{array}{l}\text { Xiang YT, et } \\
\text { al., } 2020\end{array}$ & $\begin{array}{l}\text { International journal of } \\
\text { biological sciences }\end{array}$ & A1 & 3019 & $\begin{array}{l}\text { Relatórios das } \\
\text { autoridades de } \\
\text { saúde }\end{array}$ & China/ Ásia \\
\hline $\begin{array}{l}\text { Jin YH, et } \\
\text { al., } 2020\end{array}$ & Military Medical Research & - & 103 & $\begin{array}{l}\text { Dados do Hospital } \\
\text { Zhongnan da } \\
\text { Universidade de } \\
\text { Whuan }\end{array}$ & China/Ásia \\
\hline $\begin{array}{l}\text { Ong J, et al., } \\
2020\end{array}$ & Headache & $\mathrm{A} 2$ & 158 & Questionário & $\begin{array}{l}\text { Cingapura/ } \\
\text { Ásia }\end{array}$ \\
\hline $\begin{array}{l}\text { Joob B, et } \\
\text { al., } 2020\end{array}$ & $\begin{array}{l}\text { Journal of Hospital } \\
\text { Infection }\end{array}$ & A1 & 35 & - & $\begin{array}{l}\text { Tailândia/ } \\
\text { Ásia }\end{array}$ \\
\hline $\begin{array}{l}\text { Min L, et al., } \\
2020\end{array}$ & $\begin{array}{c}\text { Jornal Chinês de } \\
\text { Tuberculose e Doenças } \\
\text { Respiratórias }\end{array}$ & - & 30 & Questionário & China/ Ásia \\
\hline $\begin{array}{l}\text { Delgado D, } \\
\text { et al., } 2020\end{array}$ & $\begin{array}{l}\text { International journal of } \\
\text { environmental research } \\
\text { and public health }\end{array}$ & $\mathrm{A} 2$ & 936 & $\begin{array}{l}\text { Escala de Likert e } \\
\text { questionário }\end{array}$ & $\begin{array}{l}\text { América } \\
\text { Latina }\end{array}$ \\
\hline $\begin{array}{l}\text { Lai J, et al., } \\
2020\end{array}$ & JAMA Network open & - & 1257 & Dados de hospitais & China/Ásia \\
\hline $\begin{array}{l}\text { Bohlken J, et } \\
\text { al., } 2020\end{array}$ & Psychiatrische Praxis & B2 & 1257 & $\begin{array}{l}\text { Revisão } \\
\text { sistemática }\end{array}$ & $\begin{array}{l}\text { Alemanha/ } \\
\text { Europa }\end{array}$ \\
\hline $\begin{array}{l}\text { Lan J, et al., } \\
2020\end{array}$ & $\begin{array}{l}\text { Journal of the American } \\
\text { Academy of Dermatology }\end{array}$ & A1 & 542 & Questionário & China/Ásia \\
\hline $\begin{array}{l}\text { Ahmed M, et } \\
\text { al., } 2020\end{array}$ & $\begin{array}{l}\text { International journal of } \\
\text { environmental research } \\
\text { and public health }\end{array}$ & $\mathrm{A} 2$ & 650 & Questionário & 30 países \\
\hline $\begin{array}{l}\text { Prevention } \\
\text { CDC, } 2020\end{array}$ & $\begin{array}{c}\text { Morbidity and Mortality } \\
\text { Weekly Report }\end{array}$ & A1 & 174 & Relatório & $\begin{array}{c}\text { Cingapura/ } \\
\text { Ásia }\end{array}$ \\
\hline $\begin{array}{l}\text { Li W, et al., } \\
2020\end{array}$ & $\begin{array}{l}\text { International Journal of } \\
\text { Biological sciences }\end{array}$ & A1 & 1563 & Relatório & China/ Ásia \\
\hline
\end{tabular}

Fonte: de Mesquita FBM, et al., 2020.

O número de profissionais de saúde infectados pela COVID-19 (Tabela 2) foi elevado em países como China. Em relação aos Estados Unidos da América (EUA), principalmente devido à falta de critérios de mitigação precoce contra a disseminação da doença (Tabela 2), o número de casos e mortes são, hoje, os maiores do mundo. O Brasil apresentou 270 óbitos, sendo 157 enfermeiros; técnicos e auxiliares; e 113 médicos. Seguindo, Rússia com 101 mortes e EUA, 549. (JIN YH, et al., 2020; MOAZZAMI B, et al., 2020; GREENBERG N, et al., 2020; MISRA A, 2020; XIANG YT, et al., 2020; JOOB B, et al., 2020; MIN L, et al., 2020; TOSTMANN A, et al., 2020; PREVENTION CDC, 2020). 
Tabela 2 - Número de profissionais de saúde infectados e mortos por COVID-19, em estudos incluídos na revisão. Juiz de Fora - MG, 2020

\begin{tabular}{cccccc}
\hline Fonte & Infectados & Mortos & Letalidade & País & Continente \\
\hline Moazzami B, et al., 2020 & 3300 & 22 & $0,6 \%$ & China & Ásia \\
Misra A, 2020 & 1716 & 6 & $0,3 \%$ & China & Ásia \\
Xiang YT, et al., 2020 & 3019 & 10 & $0,3 \%$ & China & Ásia \\
Tostmann A, et al., 2020 & 90 & - & - & Holanda & Europa \\
Jin YH, et al., 2020 & 103 & - & - & China & Ásia \\
Prevention CDC, 2020 & 9282 & 27 & $0,2 \%$ & EUA & América \\
Joob B, et al., 2020 & 40 & - & - & China & Ásia \\
Min L, et al., 2020 & 30 & 0 & $0,0 \%$ & China & Ásia \\
\hline Total & 17580 & 65 & $0,4 \%$ & - & - \\
\hline
\end{tabular}

Fonte: de Mesquita FBM, et al., 2020.

No entanto, a letalidade da doença nos profissionais de saúde oscilou de forma considerável entre os dez países com maiores números de casos, entre 0,23 até $0,81 \%$ (Tabela 3), enquanto nos estudos dos artigos considerados, esse índice atingiu 0,4\% (Tabela 2) (CONSELHO FEDERAL DE ENFERMAGEM, 2020; MINISTÉRIO DA SAÚDE DO BRASIL, 2020; CONSELHO REGIONAL DE MEDICINA DO ESTADO DE PERNAMBUCO, 2020; MINISTÉRIO DA SAÚDE DA RÚSSIA, 2020; SERVIÇO NACIONAL DE SAÚDE, 2020; PREVENTION CDC, 2020; JIN YH, et al., 2020; MOAZZAMI B, et al., 2020; GREENBERG N, et al., 2020; MISRA A, 2020; XIANG YT, et al., 2020; JOOB B, et al., 2020; MIN L, et al., 2020; TOSTMANN A, et al., 2020).

Tabela 3 - Mortes de profissionais de saúde por COVID-19 nos 10 países com maior número de casos até 20/07/20. Juiz de Fora - MG, 2020.

\begin{tabular}{ccccc}
\hline Posição & País & Mortes totais & Profissionais & Porcentagem \\
\hline $1^{\circ}$ & EUA & 139.468 & 549 & $0,39 \%$ \\
$2^{\circ}$ & Brasil & 78.772 & 270 & $0,34 \%$ \\
$4^{\circ}$ & Rússia & 12.427 & 101 & $0,81 \%$ \\
$9^{\circ}$ & Reino Unido & 45.300 & 106 & $0,23 \%$ \\
\hline Total & & 275.967 & 1.026 & $0,37 \%$
\end{tabular}

Fonte: de Mesquita FBM, et al., 2020; dados extraídos do Centers for Disease Control and Prevention (CDC), Conselho Federal de Enfermagem (COFEN), Ministério da Saúde do Brasil (MS), Conselho Regional de Medicina do Estado de Pernambuco (CREMEPE), Ministério da Saúde da Rússia e Serviço Nacional de Saúde (NHS).

Isso pode ser justificado por menor disponibilidade completa aos EPIs. Enquanto 91\% e 95\% obtiveram acesso às luvas descartáveis e desinfetante em gel para as mãos, respectivamente; apenas 32,6\% e 56,1\% lograram contato com escudos de proteção facial e a máscara N95, de acordo com estudo realizado na América Latina. Essa discrepância entre as porcentagens de acesso a EPI pode ser relacionada à inexistência de uma diretriz de saúde pública já normatizada e validada (DELGADO D, et al., 2020).

Na mesma pesquisa da América Latina, 936 profissionais entrevistados responderam através da escala de Likert, que mensurou de 1 a 10 o acesso dos participantes aos EPIs. Dos entrevistados, $899(95,1 \%)$ eram médicos, $28(2,9 \%)$ eram enfermeiros e $18(1,9 \%)$ eram profissionais de saúde aliados. Na avaliação, os participantes expressaram apoio limitado a recursos humanos, proteção à integridade física no local de trabalho e apoio das autoridades de saúde pública. Assim, esses profissionais de saúde tiveram acesso limitado aos EPIs essenciais e baixo apoio das autoridades de saúde durante a pandemia de COVID-19. Tal estudo tenta justificar o número elevado de profissionais atingidos pela pandemia (DELGADO D, et al., 2020). 
Outro aspecto importante diz respeito ao grau de conhecimento sobre o Sars-Cov-2 ser difuso entre os profissionais, uma vez que o acesso às políticas e procedimentos de segurança pessoal, segundo estudo supracitado, foi de $75,5 \%$, enquanto apenas $44,1 \%$ participaram de palestras e discussões acerca do tema. Ademais, o conhecimento sobre a prevenção da doença demostrou responder a 85,5\% dos participantes da pesquisa explicitando o não conhecimento incisivo de guidelines e a não participação em debates a respeito da COVID-19 (DELGADO D, et al., 2020).

Apesar do grande número de mortes, que pode ser observado (Tabela 2), essas não são as únicas afecções aos profissionais de saúde. Também há outros impactos físicos, emocionais e sociais, em que danos à pele, piora de cefaleia pré-existente e desenvolvimento de cefaleia por uso de EPIs contemplam de $81 \%$ a 97\% desse público (ONG J, et al., 2020; LAN J, et al., 2020)

No tocante à cefaleia associada ao uso de EPIs (Tabela 4), observou-se que a utilização prolongada desses equipamentos foi responsável pelo seu desenvolvimento, além da piora daquelas pré-existentes. Um estudo conduzido por Ong J et al. (2020); entrevistou 158 profissionais de saúde, sendo $77,8 \%$ na faixa dos 21 aos 35 anos; e 64,6\% eram enfermeiros, 32,3\% eram médicos e 3,2\% eram paramédicos. Desse grupo, $29,1 \%$ dos indivíduos já possuíam a enfermidade previamente. Oitenta e um por cento dos entrevistados desenvolveram cefaleia associada ao uso de EPIs, sendo os profissionais emergencistas os mais acometidos. Desde o início do surto de COVID-19; $91,3 \%$ dos entrevistados com diagnóstico de cefaleia primária préexistente concordaram que o aumento do uso de EPIs afetou a contenção de suas dores de cabeça, o que prejudicou seu nível de desempenho no trabalho. Em relação aos danos à pele, em um total de 542 pessoas participantes, 526 (97\%) obtiveram significativas alterações cutâneas (LAN J, et al., 2020).

Tabela 4 - Profissionais de saúde na linha de frente no combate à COVID-19 acometidos por outras enfermidades, em estudos incluídos na revisão. Juiz de Fora - MG, 2020

\begin{tabular}{cccccc}
\hline & \multicolumn{2}{c}{ Descrição } & & \multicolumn{3}{c}{ Acometidos } \\
\hline Fonte & Tipo de enfermidade & Local & N Amostral & Número & Porcentagem \\
\hline $\begin{array}{c}\text { Ong J, et al., } \\
2020\end{array}$ & $\begin{array}{c}\text { Desenvolvimento de } \\
\text { cefaleia por uso de EPI }\end{array}$ & Cingapura & 128 & 102 & $81,00 \%$ \\
$\begin{array}{c}\text { Ong J, et al., } \\
2020\end{array}$ & $\begin{array}{c}\text { Piora de cefaleia pré- } \\
\text { existente por uso de EPI }\end{array}$ & Cingapura & 46 & 42 & $91,30 \%$ \\
$\begin{array}{c}\text { Lan J, et al., } \\
2020\end{array}$ & Danos à pele & China & 542 & 526 & $97,00 \%$
\end{tabular}

Fonte: de Mesquita FBM, et al., 2020.

No que diz respeito aos impactos emocionais, foram constatados dados alarmantes sobre insônia, depressão e ansiedade entre os participantes das pesquisas, (Tabela 5). Três estudos foram conduzidos acerca do tema, sendo dois na China e um na Alemanha (LAI J, et al., 2020; BOHLKEN J, et al., 2020; LI W, 2020).

No estudo transversal, estratificado por região na China, guiado por Lai J, et al. (2020); foram coletados dados demográficos e medições de saúde mental de 1.257 enfermeiros e médicos que cuidavam de pacientes com COVID-19 em 34 hospitais, de 29 de janeiro de 2020 a 3 de fevereiro de 2020. Foi constatado que, desse grupo; $50,4 \%$ relataram depressão; $44,6 \%$ ansiedade e 34\% insônia. Entretanto, no estudo de revisão na Alemanha, guiado por Bohlken J, et al. (2020); com o mesmo número amostral, depressão e ansiedade foram observadas em apenas 2,2\% e 14,5\% dos participantes, respectivamente. Contudo, essa discrepância dos dados pode ser creditada ao fato de que não foram analisados apenas profissionais de saúde que atuam diretamente com os pacientes infectados. Por fim, em outra pesquisa conduzida na China, envolvendo 1.563 profissionais de saúde, constatou-se que mais da metade dos participantes $792(50,7 \%)$ relataram sintomas depressivos, 698 (44,7\%) de ansiedade e 564 (36,1\%), uma piora na qualidade do sono (LI W, 2020). 
Tabela 5 - Consequências da COVID-19 na saúde mental dos profissionais de saúde, em estudos incluídos na revisão. Juiz de Fora - MG, 2020.

\begin{tabular}{|c|c|c|c|c|c|c|c|c|c|c|}
\hline \multirow{3}{*}{ Fonte } & \multicolumn{10}{|c|}{ Sintomas } \\
\hline & \multirow{2}{*}{ Local } & \multirow{2}{*}{$\mathrm{N}$ amostral } & \multicolumn{2}{|c|}{ Depressão } & \multicolumn{2}{|c|}{ Ansiedade } & \multicolumn{2}{|c|}{ Insônia } & \multicolumn{2}{|c|}{ Angústia } \\
\hline & & & № & $\%$ & № & $\%$ & № & $\%$ & № & $\%$ \\
\hline $\begin{array}{l}\text { Lai J, et al., } \\
2020\end{array}$ & China & 1257 & 634 & $50,40 \%$ & 560 & $44,60 \%$ & 427 & $34,00 \%$ & 899 & $71,50 \%$ \\
\hline $\begin{array}{l}\text { Bohlken A, } \\
\text { et al., } 2020\end{array}$ & Alemanha & 1257 & 28 & $2,20 \%$ & 182 & $14,50 \%$ & - & - & - & - \\
\hline $\begin{array}{l}\text { Li W, et al., } \\
2020\end{array}$ & China & 1563 & 792 & $50,70 \%$ & 699 & $44,70 \%$ & 564 & $36,1 \%$ & - & - \\
\hline Total & & 4077 & 1454 & $35,66 \%$ & 1441 & $35,34 \%$ & 991 & $35,14 \%$ & 899 & $71,50 \%$ \\
\hline
\end{tabular}

Fonte: de Mesquita FBM, et al., 2020.

É importante salientar que outros profissionais, além de médicos e enfermeiros, têm sofrido impactos significativos com a pandemia de COVID-19. No estudo transversal de Ahmed M et al. (2020), realizado entre 10 a 17 de março de 2020, com 650 participantes de 30 países em todo o mundo, responderam a um questionário que avaliou o nível de medo ou ansiedade entre dentistas. Oitenta e sete por cento dos entrevistados tinham medo de serem infectados com COVID-19 de um paciente ou colega de trabalho. Ao tratar uma tosse ou um paciente com suspeita de infecção pela COVID-19, 90\% estavam ansiosos. Mais de $72 \%$ dos participantes se sentiram nervosos ao conversar com pacientes nas proximidades e $92 \%$ tinham medo de levar a infecção da clínica odontológica para suas famílias.

De acordo com Shacham M, et al. (2020), uma pesquisa transversal desenvolvida com 338 dentistas israelenses e higienistas dentais, que forneceram seus dados demográficos, foi avaliado o sofrimento psíquico dos participantes, mediante o estado pandêmico mundial. Cerca de $11,5 \%$ da amostra evidenciou elevado risco de estresse psicológico, dado esse representativo de um momento ainda incipiente do contexto pandêmico. Os autores afirmaram que taxas maiores são esperadas para novas pesquisas com agentes da saúde.

As considerações acerca do estudo de Guimarães F (2020), expõem a gravidade respiratória dos pacientes de COVID-19 e o risco constante de contaminação dos profissionais, sendo a rotina de trabalho nesse ambiente muito mais desgastante física e emocionalmente do que o usual para os fisioterapeutas. Dessa forma, essa pandemia evidenciou a importância do profissional de fisioterapia no tratamento intensivo, promovendo o reconhecimento da sociedade em geral e dos gestores em saúde. Outras especialidades que também podem estar em maior risco de infecção incluem anestesiologistas, que são rotineiramente expostos às secreções respiratórias em aerossol ou oftalmologistas no exame biomicroscópico com lâmpada de fenda, além de otorrinolaringologistas envolvidos em procedimentos nasais e nasofaríngeos (LI JP, et al., 2020).

\section{CONSIDERAÇÕES FINAIS}

A pandemia de COVID-19 causou um aumento rápido e volumoso da demanda por atenção à saúde. Nesse cenário, os profissionais da área se sobrecarregaram de maneira ainda não observada antes neste século. Com isso, diversos problemas emergiram tanto profissionalmente quanto pessoalmente. Entre eles, pode-se destacar: a falta de EPIs adequados, principalmente nos países em desenvolvimento; a pressão psicológica presente nesses profissionais; o desconforto decorrente da extensa utilização dos EPIs por longos períodos; o baixo índice de conhecimento científico sobre a doença por parte dos profissionais de saúde; e o distanciamento social e familiar. Em última instância, todos esses fatores são contribuintes para uma queda 
na eficácia dos atendimentos em saúde, o que se revela um paradoxo em tempos de pandemia. Diante disso, é perceptível o estado de vulnerabilidade que os diversos profissionais de saúde têm enfrentado nessa pandemia de COVID-19. Nesse sentido, a segurança destes profissionais deve ser garantida diante do estado de tensão presenciado pelos Centros de Saúde e seus trabalhadores, com o objetivo de reduzir o risco de contágio, que eleva sensações de medo e pânico generalizado. A gravidade da doença é de conhecimento mundial e os profissionais de saúde são um dos recursos mais valiosos de todos os países no combate à pandemia. Dessa forma, os estudos apresentados contribuíram para um melhor entendimento do impacto da crise humanitária desencadeada pelo coronavírus aos agentes da saúde ao redor do mundo. Vale salientar que, algumas pesquisas representaram certas limitações no que se refere aos números de mortes e pessoas infectadas, dado ao grande número de atualizações diárias e falta de padronização dos esclarecimentos prestados.

\section{REFERÊNCIAS}

1. AHMED M, et al. Fear and practice modifications among dentists to combat novel coronavirus disease (COVID-19) outbreak. International Journal of Environmental Research and Public Health, 2020; 17(8): 2821.

2. BASILE C, et al. Recommendations for the prevention, mitigation and containment of the emerging SARS-CoV-2 (COVID-19) pandemic in haemodialysis centres. Nephrology Dialysis Transplantation, 2020; 35(5): 737-741.

3. BOHLKEN J, et al. COVID-19-Pandemie: Belastungen des medizinischen Personals. Psychiatrische Praxis, 2020; 47(4): 190-197.

4. CONSELHO FEDERAL DE ENFERMAGEM. Brasil é o país com mais mortes de enfermeiros por Covid-19 no mundo. 2020. Disponível em: http://www.cofen.gov.br/brasil-e-o-pais-com-mais-mortes-de-enfermeiros-por-covid-19-nomundo-dizem-entidades_80181.html. Acesso em: 25 jun. 2020.

5. CONSELHO REGIONAL DE MEDICINA DO ESTADO DE PERNAMBUCO. Brasil ultrapassa a marca de cem médicos mortos por COVID-19, dois por dia. 2020. Disponível em: http://www.cremepe.org.br/2020/05/22/brasilultrapassa-a-marca-de-cem-medicos-mortos-por-covid-19-dois-por-dia/. Acesso em: 20 jul. 2020.

6. DELGADO D, et al. Personal Safety during the COVID-19 Pandemic: Realities and Perspectives of Healthcare Workers in Latin America. International. Journal of Environmental Research and Public Health, 2020; 17(8): 2798.

7. GREENBERG N, et al. Managing mental health challenges faced by healthcare workers during covid-19 pandemic. The BMJ, 2020; 368.

8. GUIMARÃES F. Atuação do fisioterapeuta em unidades de terapia intensiva no contexto da pandemia de COVID19. Fisioterapia em Movimento, 2020; 33.

9. HEALTH SERVICE JOURNAL. Exclusivo: mortes de funcionários do NHS da COVID-19 analisadas. 2020. Disponível em: https://www.hsj.co.uk/exclusive-deaths-of-nhs-staff-from-covid-19-analysed/7027471.article. Acesso em: 20 jul. 2020.

10. HUH S. How to train health personnel to protect themselves from SARS-CoV-2 (novel coronavirus) infection when caring for a patient or suspected case. Journal of Educational Evaluation for Health Professions, 2020; 17.

11. ITELMAN E, et al. Clinical characterization of 162 COVID-19 patients in Israel: preliminary report from a large tertiary center. The Israel Medical Association Jornal, 2020; 22(5).

12. JIN YH, et al. Perceived infection transmission routes, infection control practices, psychosocial changes, and management of COVID-19 infected healthcare workers in a tertiary acute care hospital in Wuhan: a cross-sectional survey. Military Medical Research, 2020; 7: 1-13.

13. JOOB B, et al. COVID-19 in medical personnel: observation from Thailand. Journal of Hospital Infection, $2020 ; 10$.

14. KAWCHUK G, et al. The use of internet analytics by a Canadian provincial chiropractic regulator to monitor, evaluate and remediate misleading claims regarding specific health conditions, pregnancy, and COVID-19. Chiropractic \& Manual Therapies, 2020; 28: 1-7.

15. KHAN S, et al. The spread of novel coronavirus has created an alarming situation worldwide. Journal of Infection and Public Health, 2020; 13(4): 469.

16. KISELY S, et al. Occurrence, prevention, and management of the psychological effects of emerging virus outbreaks on healthcare workers: rapid review and meta-analysis. The BMJ, 2020; 369.

17. KOH D. Occupational risks for COVID-19. Occupational Medicine, 2020; 70(1): 3-5.

18. LAI J, et al. Factors associated with mental health outcomes among health care workers exposed to coronavirus disease 2019. Jama Network Open, 2020; 3(3): e203976-e203976.

19. LAN J, et al. Skin damage among health care workers managing coronavirus disease-2019. Journal of the American Academy of Dermatology, 2020; 82(5): 1215-1216.

20. LANCET T. COVID-19: protecting health-care workers. The Lancet, 2020; 395(10228): 922.

21. LI JP, et al. Preparedness among ophthalmologists: during and beyond the COVID-19 pandemic. American Academy of Ophthalmology, 2020; 127(5): 569-572.

22. LI W, et al. Progression of mental health services during the COVID-19 outbreak in China. International journal of biological sciences, 2020; 16(10): 1732. 
23. LICCIARDI F, et al. COVID-19 and what pediatric rheumatologists should know: a review from a highly affected country. Pediatric Rheumatology Online Journal, 2020; 18(1): 1-7.

24. LITTLE P, et al. Reducing risks from coronavirus transmission in the home the role of viral load. The BMJ, $2020 ; 369$.

25. MARK A, et al. Essential new information for the clinical recognition of COVID-19 infection and the prevention possibilities of healthcare personnel working in the head and neck region. Orvosi Hetilap, 2020; 161(17): 660-666.

26. MIN L, et al. Análise das características clínicas de 30 novas pneumonias por coronavírus na equipe médica. Jornal Chinês de Tuberculose e Doenças Respiratórias, 2020; 43.

27. MINISTÉRIO DA SAÚDE DA RÚSSIA. O Ministério da Saúde anunciou pela primeira vez o número de mortes por médicos com coronavírus, 2020. Disponível em: https://xn--80aesfpebagmfblc0a.xn--p1ai/news/20200526-1709.html. Acesso em: 20 jul. 2020.

28. MINISTÉRIO DA SAÚDE DO BRASIL. Coronavírus Brasil, 2020. Disponível em: http://www.cremepe.org.br/2020/05/22/brasil-ultrapassa-a-marca-de-cem-medicos-mortos-por-covid-19-dois-pordial. Acesso em: 20 jul. 2020.

29. MISRA A. Doctors and healthcare workers at frontline of COVID 19 epidemic: Admiration, a pat on the back, and need for extreme caution. Diabetes \& Metabolic Syndrome, Netherlands, 2020.

30. MOAZZAMI B, et al. COVID-19 and telemedicine: Immediate action required for maintaining healthcare providers wellbeing. Journal of Clinical Virology, 2020; 126: 104345.

31. ONG J, et al. Headaches Associated With Personal Protective Equipment-A Cross-Sectional Study Among Frontline Healthcare Workers During COVID-19. Headache: The Journal of Head and Face Pain, 2020; 60(5): 864-877.

32. ORNELL F, et al. The impact of the COVID-19 pandemic on the mental health of healthcare professionals. Cadernos de Saúde Pública, 2020; 36: e00063520.

33. PREVENTION CDC. Characteristics of Health Care Personnel With COVID-19. Morbidity and Mortality Weekly Report, 2020; 69(15): 477-481.

34. RANNEY ML, et al. Critical supply shortages the need for ventilators and personal protective equipment during the Covid-19 pandemic. New England Journal of Medicine, 2020; 382(18): e41.

35. SHACHAM M, et al. COVID-19 Factors and Psychological Factors Associated with Elevated Psychological Distress among Dentists and Dental Hygienists in Israel. International Journal of Environmental Research and Public Health, 2020; 17(8): 2900.

36. TOSTMANN A, et al. Strong associations and moderate predictive value of early symptoms for SARS-CoV-2 test positivity among healthcare workers, the Netherlands. Eurosurveillance, 2020; 25(16): 2000508.

37. VERBEEK JH, et al. Personal protective equipment for preventing highly infectious diseases due to exposure to contaminated body fluids in healthcare staff. Cochrane Database of Systematic Reviews, 2020; (4).

38. WORLD HEALTH ORGANIZATION. WHO Coronavirus Disease (COVID-19) Dashboard. 2020. Disponível em: https://covid19.who.int/. Acesso em: 20 jul. 2020.

39. XIANG YT, et al. Tribute to health workers in China: A group of respectable population during the outbreak of the COVID-19. International journal of biological sciences, 2020 16(10): 1739. 